Military Technical College

Kobry El-Kobbah, Cairo, Egypt

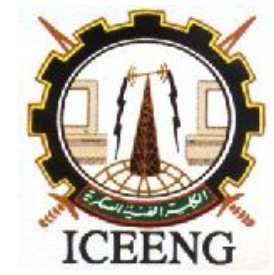

$9^{\text {th }}$ International Conference on Electrical Engineering

ICEENG 2014

\title{
Estimation of IEEE802.11a Signal Parameters using Power Spectrum Properties
}

\author{
By \\ Hazem M. Sakr* Ehab M. Shaheen* Sherif Elgamel* Ahmed S. Elmahdy**
}

\section{$\underline{\text { Abstract: }}$}

In this paper, the orthogonal frequency division multiplexing (OFDM)- based Wi-Fi IEEE802.11a signal parameters (bandwidth, subcarrier spacing, and the number of subcarriers) are experimentally estimated over a band of signal to noise ratio (SNR) values. The estimation of the considered OFDM system parameters is blindly done with the aid of OFDM power spectrum properties. The estimation is evaluated under the assumption of additive white Gaussian noise (AWGN) and frequency selective multipath fading channels. The proposed estimation technique based on power spectrum processing technique which takes full advantage of the cryptic period of the OFDM signal. It was shown that the proposed method is applicable for medium SNR and it requires low computational complexity.

\section{Keywords:}

Blind estimation, OFDM signal, IEEE802.11a, and multi-path fading channel.

* $\quad$ Egyptian Armed Forces

** German University in Cairo (GUC) 


\section{Introduction:}

OFDM has been selected as a candidate for many civilian and military applications. This is because of its robustness against frequency selective fading channel, and its capability of transmitting high data rates over wireless channel $[1,2]$.

OFDM was widely employed in the recent decades as a modulation scheme that adopts multiple carriers with equal frequency intervals to form high data rate transmission $[1,2]$. OFDM based Wi-Fi IEEE802.11a is a multi-carrier modulation scheme, which is capable of transmitting data at rate up to 54Mbps when using 64-QAM [3].

The well-known of communication signal parameters such as signal bandwidth, spacing between subcarriers, and the number of subcarriers are very important in civilian and military applications such as cognitive radio (CR). CR is an intelligent device, which has to be aware of its propagation environment and adapt itself by making real time changes to its operating parameters. Thus, it is necessary to develop a blind OFDM parameters estimation algorithm to estimate these parameters.

Detection of spread spectrum signals was developed using power properties of the signal over AWGN channel in $[4,5]$. In [6], an approximate method to detect spread spectrum signal depending on its power over flat fading channel was driven. The authors in $[8,9]$ et. al. use the autocorrelation function periodicity property of OFDM signal in time domain to analyze and intercept this signal depending on second order cyclo-stationarity.

In this paper, an experimental method for blind estimation of OFDM based Wi-Fi IEEE802.11a signal parameters using its power spectrum properties is presented. This estimation is done for OFDM signal at different SNR values.

The idea of the proposed method is based on using the signal power spectrum width to calculate the signal bandwidth, while the separation between two peaks of the square of the autocorrelation function is used to calculate the subcarriers spacing. The number of subcarriers is evaluated by investigating the obtained data of signal bandwidth and subcarriers spacing. The proposed method depends on the periodicity of OFDM signal spectrum in frequency domain and the properties of the autocorrelation function in time domain.

The paper is organized as follow. The OFDM signal model is presented in section 2. In section 3 an analysis of the power spectrum of OFDM signal over AWGN and multipath fading channels is proposed. In section 4 a computer based simulation and results are presented. Finally, section 5 concludes the presented work. 


\section{OFDM signal model :}

The OFDM signal transmitted over wireless channel can be written as [10]

$$
x(t)=\frac{1}{K} \sum_{k=1}^{K} \mathbf{A}_{k} \mathbf{e}^{\mathbf{j}\left(2 \pi k \Delta f t+\phi_{k}\right)}
$$

where $\mathrm{K}$ is the number of subcarriers, $\Delta f$ is the frequency separation between two adjacent subcarriers equal to $\frac{\mathbf{1}}{T_{s}}\left(T_{s} .\right.$. OFDM symbol period), $\mathbf{A}_{k}$ and $\phi_{k}$ are the amplitude and the phase of $k^{\text {th }}$ subcarrier.

At the receiver, the signal is affected by multi-path time dispersive channel thus the OFDM signal can be written as $[11,12]$

$$
x(t)=\frac{1}{K} \sum_{n=1}^{\mathbf{N}} \sum_{k=1}^{\mathbf{K}} \mathbf{A}_{k} \mathbf{e}^{\mathbf{j}\left(2 \pi k \Delta f\left(\mathbf{t}-\tau_{n}\right)+\phi_{k}\right)}{ }_{h\left(\tau_{n}\right)},
$$

where $\boldsymbol{h}\left(\tau_{n}\right)$ is the channel response due to multipath, $\tau_{n}$ is the delay of the $n^{\text {th }}$ path. And $N$ is the number of paths.

\section{OFDM signal interception:}

OFDM based IEEE802.11a signal parameters are experimentally estimated under the assumption of the cases: AWGN channel and multi-path time dispersive channel.

From equation (1) and the definition of the Fourier Transform, it is noticed that $\mathbf{A}_{k} \mathrm{e}^{\mathbf{j} \mathbf{\phi}_{k}}$ is the signal definition in frequency domain, thus the OFDM signal can be introduced by Fourier transform for one symbol period as [14]

$$
x(f)=\frac{2 \pi}{K} \sum_{k=1}^{K} A_{k} \delta(f-k \Delta f)
$$

where $\delta$ is the dirac delta function.

and the signal power spectrum is

$$
P(f)=x(f) \cdot x^{H}(f)=\mid[x(f)]^{2},
$$




$$
=\left(\frac{2 \pi}{K}\right)^{2} \sum_{k=1}^{K} A_{k}^{z} \delta(f-k \Delta f)
$$

where $H$ represents the Hermitian of the vector signal.

If many symbol periods considered thus the signal's spectrum is written as [14]

$$
\begin{aligned}
x_{o}(f) & =x(f) \otimes x_{k}(f) \\
& =\frac{2 \pi}{K} \sum_{k=1}^{K} A_{k} \delta(f-k \Delta f) \otimes x_{k}(f) \\
& =\frac{2 \pi}{K} \sum_{k=1}^{K} A_{k} x_{k}(f-k \Delta f)
\end{aligned}
$$

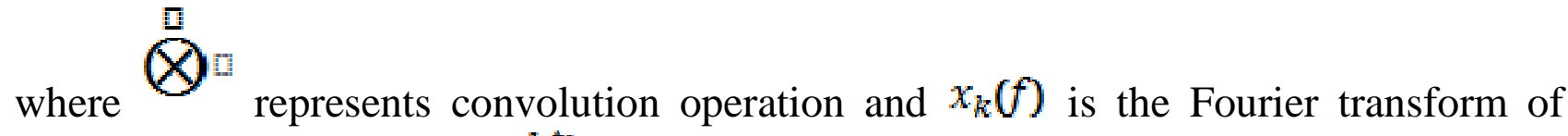
multiple data symbols of the $k^{\text {th }}$ subcarrier.Then the power spectrum can be written as

$$
\begin{aligned}
P_{o}(f) & =x_{o}(f) \cdot x_{o}^{H}(f) \\
& =\left(\frac{2 \pi}{K}\right)^{2} \sum_{k=1}^{K} A_{k}^{2}\left|x_{k}(f-k \Delta f)\right|^{2}
\end{aligned}
$$

From equation (6), it can be noticed that the power spectrum of the OFDM signal is periodic with period equal to $\boldsymbol{\Delta} f$. After detecting the signal power spectrum, apply the inverse Fourier transform and square. The result is a representation to the square of the autocorrelation function of the signal.

$$
\begin{aligned}
& R(\tau)=I F F T\left[P_{o}(f)\right] \\
& x(\tau)=\left.I I F F T\left[P_{o}(f)\right]\right|^{2}
\end{aligned}
$$

This leads to

$$
x(\tau)=c|R(-\tau)|^{2}=c|R(\tau)|^{2}
$$

where $c$ is a constant, and $R(\tau)$ is the autocorrelation function of the OFDM signal which is even function. It is seen that the power spectrum processing is the square of the modulus of the autocorrelation function. 
Case 1: AWGN Channel

The OFDM signal model over AWGN channel can be written as

$$
r(t)=x(t)+n(t)
$$

where $n(t)$ is the AWGN

The power of the received signal is

$$
P_{r}(f)=P_{o}(f)+P_{n}(f)
$$

where $P_{n}(f)$ is the noise power spectrum equals to $N_{0}$. The processed spectrum is written as [14]

$$
\begin{aligned}
& x\left(t_{r}\right)=\|\left. I F F T\left[P_{r}(f)\right]\right|^{2} \\
&=\left.I I F F T\left[P_{o}(f)+N_{0}\right]\right|^{2} \\
&=\|\left. I F F T\left[P_{o}(f)\right]\right|^{2}+\left|I F F T\left[N_{o}\right]\right|^{2}+2\left|I F F T\left[P_{o}(f)\right] I F F T\left[N_{\circ}\right]\right| \\
&=\left|N_{0.2 \pi} \cdot \delta\left(t_{r}\right)\right|^{2}+c\left|R_{o}\left(t_{r}\right)\right|^{2}+\left|2 N_{0.2 \pi} \cdot R_{o}(0) . \delta\left(t_{r}\right)\right|
\end{aligned}
$$

where $R_{o}\left(t_{r}\right)$ is the autocorrelation function of the signal and $c$ is a constant. The noise spectrum processing has only one peak at zero, and the OFDM signal autocorrelation function has narrow pulses appear periodically at $T_{s}$.

Figure (1) shows the power spectrum processing over AWGN channel at $\mathrm{SNR}=3 \mathrm{~dB}$. 


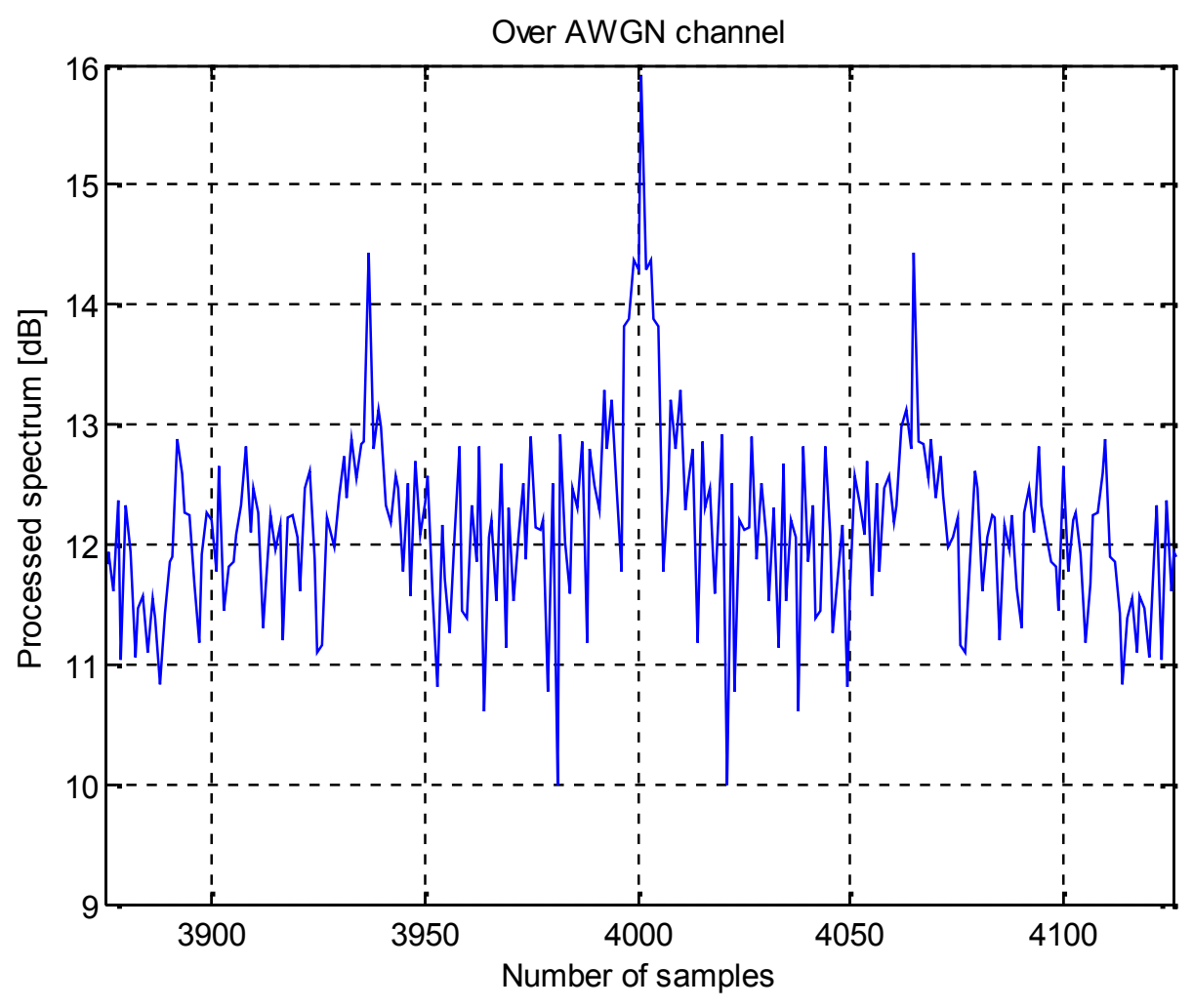

Fig. (1) Power spectrum processing over AWGN channel.

In figure (1), the processed spectrum has a peak at the center due to the noise power and has two side lobes at $R(-\tau)$ and $R(\tau)$.

Case 2: Multipath Fading Channel

Multipath in a communication channel causes signal fading, where the received signal is the sum of multiple replicas of the original signal. It can be represented as

$$
x_{f}(t)=\sum_{n=1}^{N} x(t) h\left(t-\tau_{n}\right)
$$

For simplicity let $N=2$, signal from path number " 1 " is $x(t)$, and signal from path "2" is $\boldsymbol{h} x(t+\Delta t) e^{j \mathbf{z} \pi f \Delta t}$

where $\boldsymbol{h}$ represents the attenuation coefficient between paths, and $\Delta t$ represents the delay between paths. $\Delta t$ is considered very small, which can be approximated as $x(t+\Delta t) \cong x(t)$. 


$$
x_{f}(t)=x(t)\left(1+h e^{j 2 \pi f \Delta t}\right)
$$

The channel transfer function can be written as

$$
\begin{aligned}
H(f) & =\frac{x_{f}(t)}{x(t)} \\
& =1+h e^{j \pi \pi f \Delta t}
\end{aligned}
$$

and the channel amplitude is

$$
\begin{aligned}
A(f) & =|H(f)| \\
& =\sqrt{1+h^{2}+2 h \cos (2 \pi f \Delta t)}
\end{aligned}
$$

Now processing the signal as represented in equation (11)

$$
\begin{aligned}
x_{f}\left(t_{e}\right) & =\left|I F F T\left[|x(f)|^{2}|H(f)|^{2}\right]\right|^{2} \\
& =\mid I F F T\left(\left.\left|x_{o}(f)\right|^{2}\left[\left(1+h^{2}\right)+2 h \cos (2 \pi f \Delta t)\right]\right|^{2}\right. \\
& =\left(1+h^{2}\right) c\left|R_{o}\left(\tau_{n}\right)\right|^{2}+h^{2} c\left[\left|R_{o}\left(\tau_{n}-\Delta t\right)\right|^{2}+\left|R_{o}\left(\tau_{n}+\Delta t\right)\right|^{2}\right]
\end{aligned}
$$

Figure (2) shows, the power spectrum processing over fading channel with $\mathrm{SNR}=3 \mathrm{~dB}$ according to equation (17). 


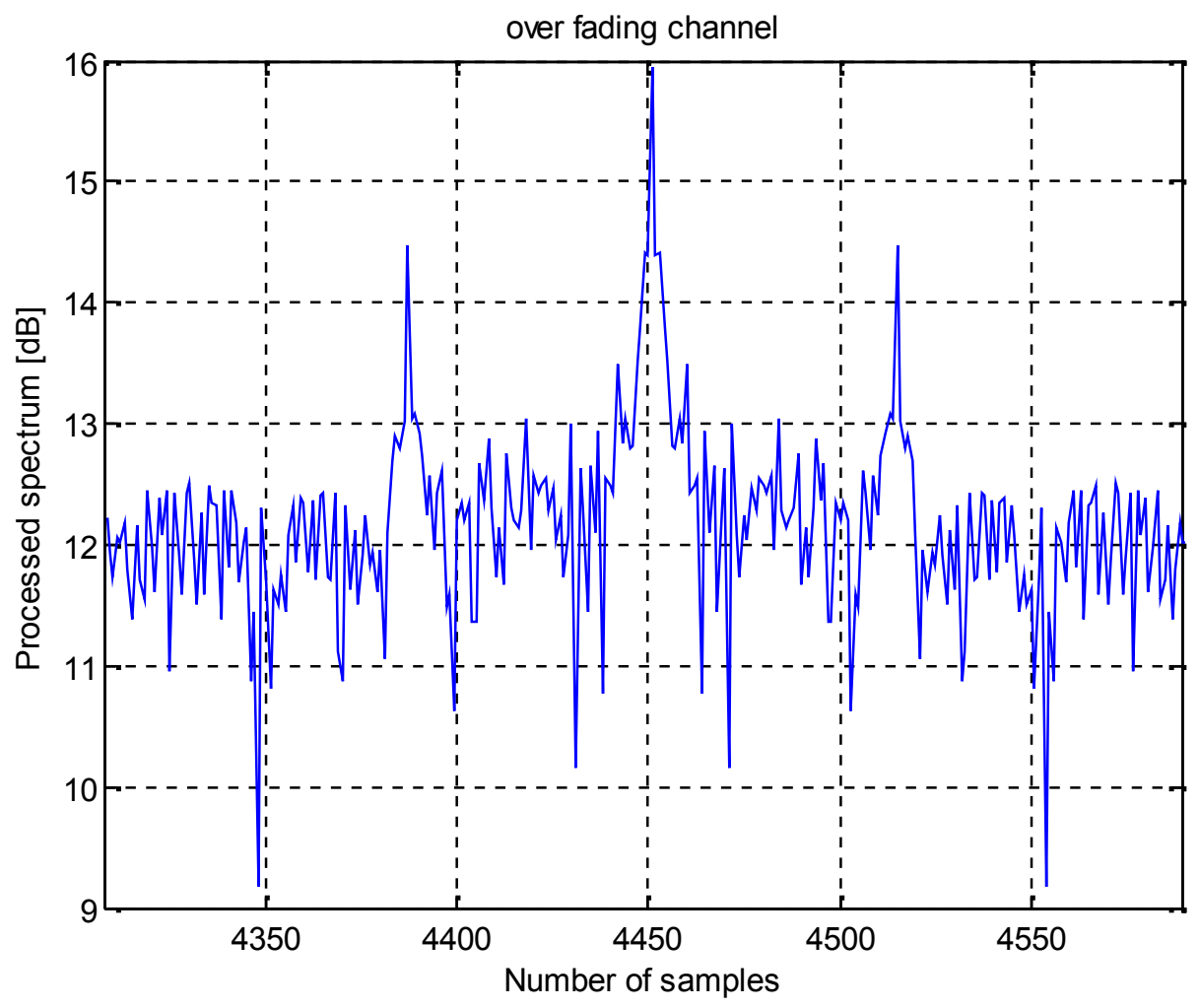

Fig.(2) Power spectrum processing over fading channel.

It is noticed that the signal bandwidth in case of fading channel becomes wider than in case of AWGN channel due to time delay of the signal replicas $\left(\tau_{n}-\Delta t\right),\left(\tau_{n}+\Delta t\right)$.

\section{Simulation and Signal Parameters Estimation:}

In this section, the OFDM parameters -based IEEE802.11a- will be estimated experimentally using power spectrum and processed power spectrum properties. This parameters estimation is averaged over 100 runs for different values of SNR.

The standard parameters of OFDM signal are given in table (1).

\begin{tabular}{|c|c|c|c|}
\hline OFDM signal & Bandwidth & $\begin{array}{c}\text { Number of } \\
\text { subcarriers }\end{array}$ & Subcarriers spacing \\
\hline IEEE802.11a & $20 \mathrm{MHz}$ & 64 & $312.5 \mathrm{KHz}$ \\
\hline
\end{tabular}

Table (1) The standard OFDM signal parameters 
Simulation was executed using the computer based program (Matlab 2013), to estimate experimentally the parameters of the OFDM based IEEE802.11a Wi-Fi signal.

The simulation parameters are set as follows according to the IEEE802.11a standard: the OFDM bandwidth is $20 \mathrm{MHz}$ with an occupied bandwidth of $16.6 \mathrm{MHz}$, the number of subcarriers are 64, where 48 of them represent the useful data, 4 are pilot tones and 12 are set to zero with a carrier separation of $0.3125 \mathrm{MHz}$. The Symbol duration is 4 microseconds, which includes a guard interval of 0.8 microseconds. The time domain signal is generated by taking an Inverse Fast Fourier transform (IFFT). The Sampling frequency is $20 \mathrm{MHz}$.

a) Bandwidth estimation:

The estimation of the signal bandwidth will be done for different values of SNR (-3 up to $5 \mathrm{~dB}$ ). By using a periodogram algorithm in order to estimate the average power spectrum [15], its length is L. The two end point frequency positions of the power spectrum are $(a, b)$.

Then the signal bandwidth $(B . W)$ is

$$
B . W=\text { round }\left[(b-a) \cdot \frac{f_{s}}{L}\right]
$$

where $f_{s}$ is the sampling frequency. This is shown in figure (3)

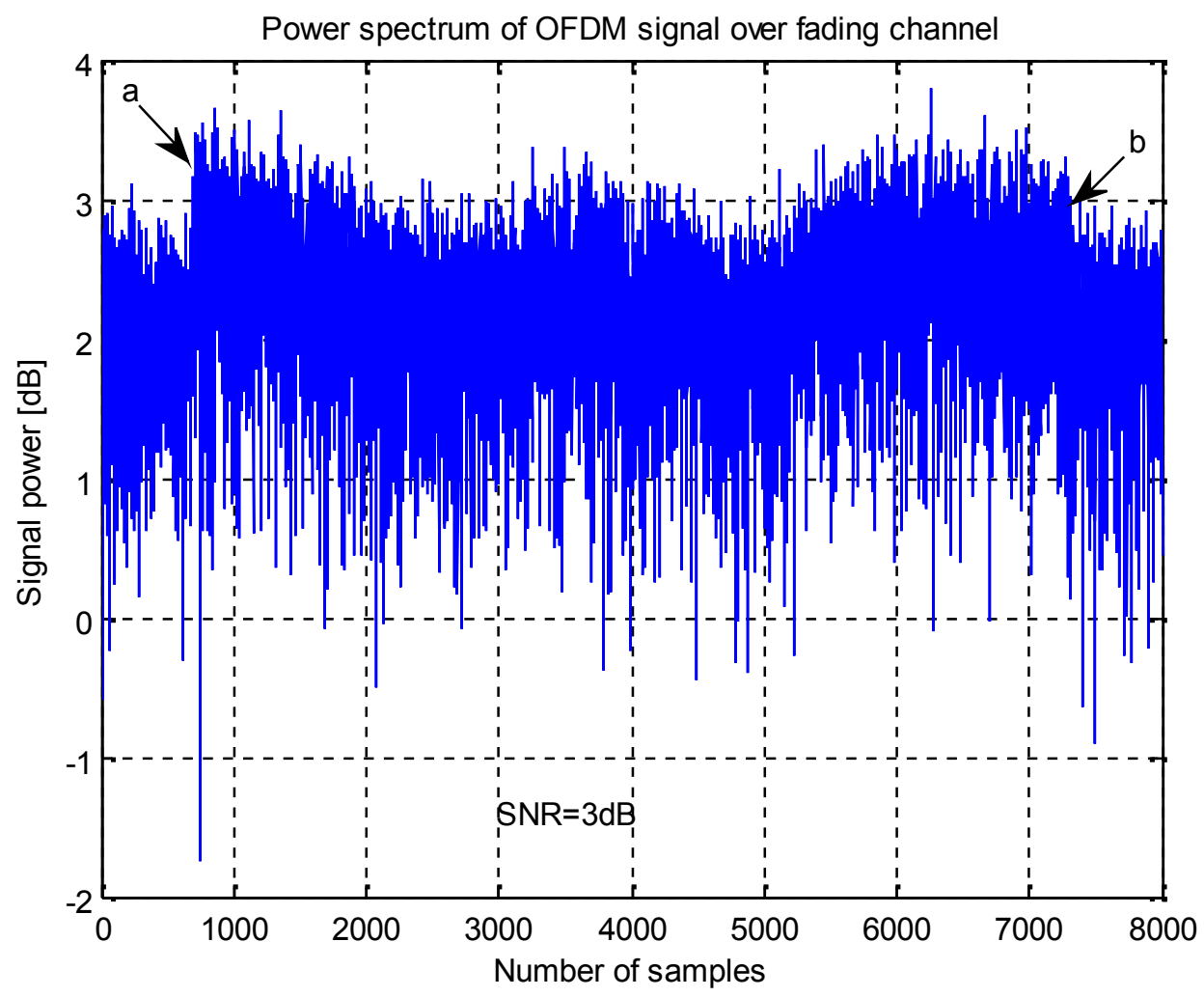


Fig.(3) Power spectrum of OFDM signal

In figure (3), the two end point frequency positions $a$, and $b$ are declared. A fading channel with 6 paths is applied. For a SNR less than $2 \mathrm{~dB}$ the signal was merged in the noise and the required data was not obtained to determine the signal bandwidth. This is shown in table (2).

b) Subcarriers spacing and Number of subcarriers:

The signal subcarriers spacing estimation will be done for different values of SNR ( -3 up to $5 \mathrm{~dB}$ ). Measure the spacing between two adjacent peaks $D$ for the processed spectrum as shown in figure (4), then calculate the subcarrier spacing as

$$
\Delta f=\frac{f_{s}}{D}
$$

from the signal bandwidth and the spacing between subcarriers, the number of subcarriers is

$$
K \cong \operatorname{round} \frac{B \cdot W}{\Delta f}
$$

For SNR less than $-2 \mathrm{~dB}$ the processed signal was merged in the noise and the required data was not obtained to determine the subcarriers spacing. This is shown in table (2). 


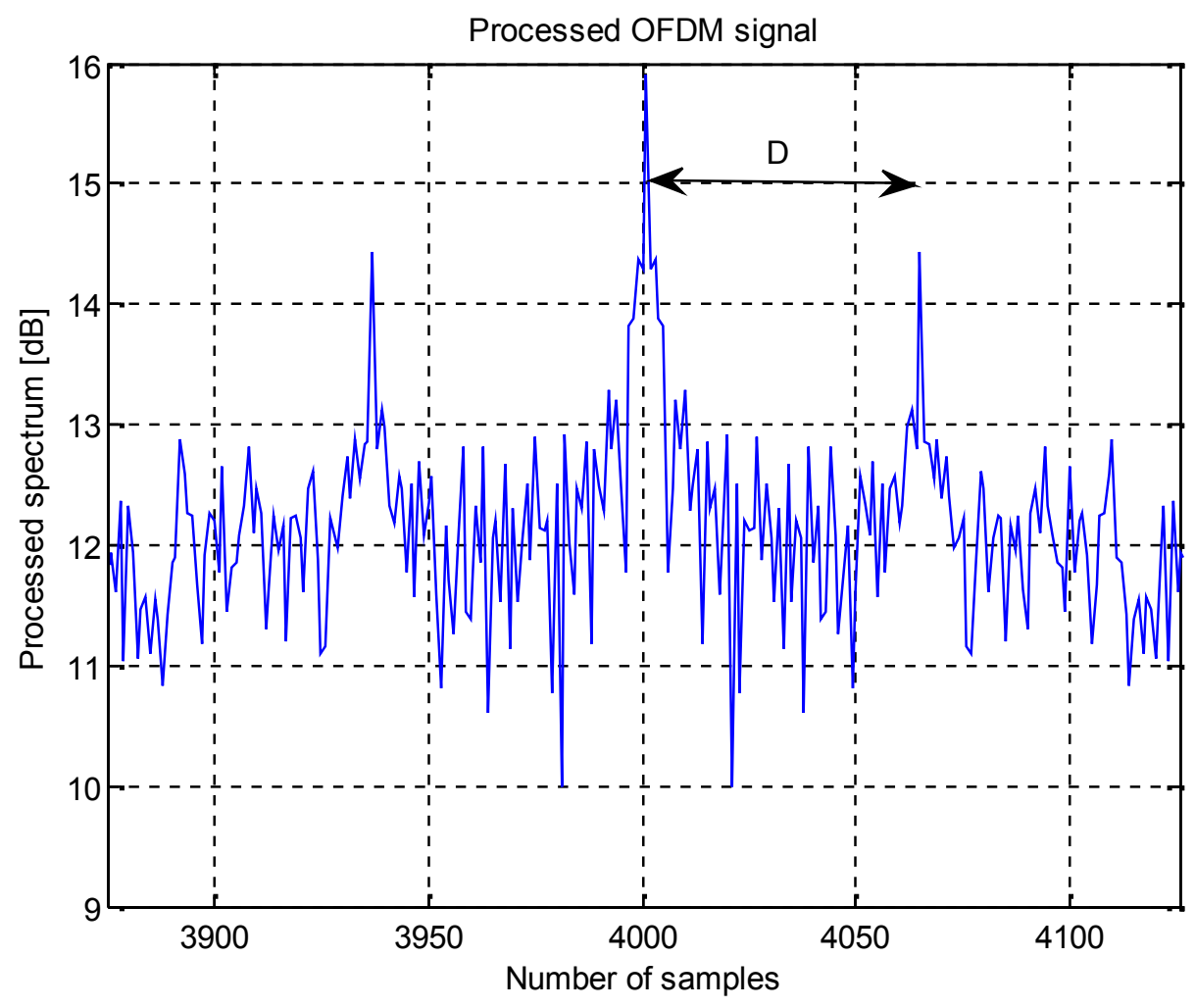

Fig. (4) Power spectrum processing of OFDM signal.

Processing the signal with SNR less than $1 \mathrm{~dB}$ make the detection of the signal power spectrum not possible, then the calculation of the signal band-width is not done. But the detection of the square of the autocorrelation function can be recognized, this is fulfilled for $\mathrm{SNR}=-2 \mathrm{~dB}$. Thus the subcarrier spacing could be estimated.

\begin{tabular}{|c|c|c|c|}
\hline SNR $(\mathrm{dB})$ & $\begin{array}{c}\text { Bandwidth } \\
(\mathrm{MHz})\end{array}$ & $\begin{array}{c}\text { Subcarriers spacing } \\
(\mathrm{KHz})\end{array}$ & $\begin{array}{c}\text { Number of } \\
\text { subcarriers }\end{array}$ \\
\hline-3 & - & - & - \\
\hline-2 & - & 312.5 & - \\
\hline 1 & - & 312.5 & - \\
\hline 2 & 20 & 312.5 & 64 \\
\hline 3 & 20 & 312.5 & 64 \\
\hline
\end{tabular}

Table (2). The estimated OFDM signal parameters. 


\section{Conclusion:}

In this paper, OFDM based IEEE802.11a signal could be intercepted by processing power spectrum of the signal. This method is applicable for both AWGN channel and multipath channel. It can eliminate single carrier signals by periodic detection of the spectrum processing of the OFDM signal. The parameters of the OFDM signal (signal bandwidth, subcarriers spacing and number of subcarriers) are estimated for medium SNR. Simulation results were done using $S N R=(-3$ up to $5 \mathrm{~dB})$ for both $A W G N$ and multipath channels. The signal band-width could be estimated for SNR greater than 2 $\mathrm{dB}$, while the subcarrier spacing could be estimated for SNR more than -2dB.

\section{References:}

[1] R. V. Nee and R. Prasad, OFDM Wireless Multimedia Communications. ArtechHouse, 2000.

[2] T.Weiss and F. K. Jondral, "Spectrum pooling: an innovative strategy for theenhancement of spectrum efficiency," IEEE Commun. Mag., vol. 43, no. 3, pp. S8-S14, Mar. 2004.

[3] Richard van Nee, "A new OFDM standard for high rate wireless LAN in the $5 \mathrm{GHz}$ band," Proceedings Vehicular Technology Conference, Volume 1, pp.258-262, September 19-22, 1999.

[4] Don Torrieri, Principles of spread-spectrum communication systems, 2005.

[5] Stephen A. Stoops, David Norman and Glenn E. Prescott, "Simulation of Radiometer for detection of spread-spectrum signals",1990

[6] Khairy El Barbary, Hossam El-Din Abou Bakr, Ahmed El-Sayed El Mahdy, Hazem M. Kamal, "Detection of direct sequence over flat fading channel" $7^{\text {th }}$ International Conference on Electrical Engineering ICEENG 2010.

[7] E Axell, G Leus, EG Larsson, Overview of spectrum sensing for cognitive radio, in 2nd International Workshop on Cognitive Information Processing (Elba, 2010), pp. 322-327.

[8] M Shi, Y Bar-Ness, W Su, Blind OFDM systems parameters estimation for software defined radio, in IEEE Symposium on New Frontiers in Dynamic Spectrum Access Networks (Dublin, 2007), pp. 119-122.

[9] A. Punchihewa, Vijay K. Bhargava, and Charles Despins, Blind Estimation of OFDM Parameters in Cognitive Radio Networks, IEEE Transations on Wireless Communications, Vol. 10, No. 3, MARCH 2011.

[10] A. Gardner, Cyclostationarity in Communications and Signal Processing, IEEE Press, 1994.

[11] Zhuo Sun*, Ruzhe Liu and Wenbo Wang, Joint time-frequency domain 
cyclostationarity-based approach to blind estimation of OFDM transmission parameters, Sun et al. EURASIP Journal on Wireless Communications and Networking 2013, 2013:117

[12] K. Po and J. Takada, Signal detection for analog and digital TV signals for cognitive radio, IEICE Technical Report, SR2006-54, November 2006.

[13] Kimtho PO $\dagger$ and Jun-ichi TAKADA, Signal Detection Method based on Cyclostationarity for Cognitive Radio, Tokyo Institute of Technology 2-12-1 O-okayama, Meguro-ku, Tokyo, 152-8550 Japan.

[14] ZHANG Hai-ying YUAN Chao-wei, A Method for Blind Detection of OFDM Signal Based on Power Spectrum Reprocessing, Eighth ACIS International Conference on Software Engineering, Artificial Intelligence, Networking, and Parallel/Distributed Computing 2007.

[15] Peter D. Welch, The use of Fast Fourier Transform for the estimation of power spectra: A method based on time averaging over short, modified periodograms, IEEE Trans. Audio and electroacoust., vol. AU-15, pp. 70-73, june 1967. 